\title{
Predicting Risky Drinking Outcomes Longitudinally: What Kind of Advance Notice Can We Get?
}

\author{
Robert A. Zucker, Maria M. Wong, Duncan B. Clark, Kenneth E. Leonard, \\ John E. Schulenberg, Jack R. Cornelius, Hiram E. Fitzgerald, Gregory G. Homish, \\ Alicia Merline, Joel T. Nigg, Patrick M. O'Malley, and Leon I. Puttler
}

\begin{abstract}
This paper summarizes the proceedings of a symposium presented at the 2005 Research Society on Alcoholism meeting in Santa Barbara, California, that spans the interval from toddlerhood to early middle adulthood and addresses questions about how far ahead developmentally we can anticipate alcohol problems and related substance use disorder and how such work informs our understanding of the causes and course of alcohol problems and alcohol use disorder. The context of these questions both historically and developmentally is set by Robert Zucker in an introductory section. Next, Maria Wong and colleagues describe the developmental trajectories of behavioral and affective control from preschool to early adolescence in a high risk for alcoholism longitudinal study and demonstrate their ability to predict alcohol and drug outcomes in adolescence. Duncan Clark and Jack Cornelius follow with a report on the predictive utility of parental disruptive behavior disorders in predicting onset of alcohol problems in their adolescent offspring in late adolescence. Next, Kenneth Leonard and Gregory Homish report on adult development study findings relating baseline individual, spouse, and peer network drinking indicators at marriage onset that distinguish different patterns of stability and change in alcohol problems over the first 2 years of marriage. In the final paper, John Schulenberg and colleagues, utilizing national panel data from the Monitoring the Future Study, which cover the 18- to 35-year age span, show how trajectories of alcohol use in early adulthood predict differential alcohol abuse and dependence outcomes at age 35. Finally, Robert Zucker examines the degree to which the core symposium questions are answered and comments on next step research and clinical practice changes that are called for by these findings.
\end{abstract}

Key Words: Longitudinal Studies, Predicting Alcohol Problems, High-Risk Research, EarlyOnset Drinking.

\section{INTRODUCTION}

$\mathrm{O}$ VER THE PAST generation, a number of major longitudinal studies have been tracking stability and change in alcohol involvement, in risk, and in correlates of

From the Department of Psychiatry, University of Michigan Addiction Research Center, Ann Arbor, Michigan (RAZ, AM, LIP); the Department of Psychology, Idaho State University, Pocatello, Idaho $(M M W)$; the Departments of Psychiatry and Pharmaceutical Sciences and the Center for Education and Drug Abuse Research, University of Pittsburgh, Pittsburgh, Pennsylvania (DBC, JRC); the Research Institute on Addictions, Buffalo, New York (KEL, GGH); the Institute for Social Research, University of Michigan, Ann Arbor, Michigan (JES, AM, PMO); and the Department of Psychology, Michigan State University, East Lansing, Michigan (HEF, JTN).

Received for publication September 30, 2005; accepted October 17, 2005.

The work of RAZ, MMW, and colleagues was supported by NIAAA Grants $R 37$ AA07065 and R01 AA12217, of DBC and JRC by Grants K02 AA00291 and NIDA Grants P50 DA05605 and R01-DA14635, of KEL and GGH by NIAAA Grant R37 AA09922, and of JES and colleagues by NIDA Grant R01 DA01411 (The Monitoring the Future Study).

Reprint requests: Robert A. Zucker, University of Michigan Addiction Research Center, 2025 Traverwood Dr. Ste A, Ann Arbor, MI 48105; Fax: (734) 998-7992; E-mail: zuckerra@umich.edu

Copyright (C) 2006 by the Research Society on Alcoholism.

DOI: $10.1111 /$ j.1530-0277.2006.00033.x course and have come to the point where 2 questions can be asked as follows: How far ahead can we anticipate alcohol problems and related substance use disorder? What can this work tell us about the causes and the course of disorder, long before the problems and symptomatology show up? This symposium addresses these questions in a group of longitudinal studies that span the interval from toddlerhood to early middle adulthood. Studies presented by Maria Wong, Duncan Clark, and colleagues involve high-risk research designs. The Wong et al. paper describes the developmental trajectories of behavioral and affective control from preschool to early adolescence and shows their ability to predict alcohol and drug outcomes in adolescence. The Clark and Cornelius paper, spanning the interval from late childhood to late adolescence, shows how parental disruptive behavior disorder, an index of their own childhood dysregulation, predicts age of onset of alcohol problems in their children during adolescence. The centrality of this variable as a marker of the underlying diathesis is suggested by the fact that parental alcohol use disorder (AUD) does not add any additional variance to the predictive equation. The Leonard and Homish study shifts the life cycle focus to early marriage and reports 
on baseline individual, spouse, and peer predictors at marriage onset that distinguish different patterns of stability and change in alcohol problems over the first 2 years of marriage. The Schulenberg et al. paper reports on national panel data that cover the 18- to 35-year age span and shows how trajectories of alcohol use in early adulthood predict differential alcohol abuse and dependence outcomes at age 35. Finally, Robert Zucker discusses the commonalities among these studies, examines the degree to which the core symposium questions are answered, and comments on next step research and clinical practice changes that are called for by these findings.

\section{EFFECTS OF BEHAVIORAL CONTROL AND RESILIENCY IN THE ONSET OF ALCOHOL AND ILLICIT DRUG USE: TRAJECTORY RELATIONSHIPS FROM PRESCHOOL TO ADOLESCENCE}

\section{Maria M. Wong, Joel T. Nigg, Leon. I. Puttler, Hiram E. Fitzgerald, and Robert A. Zucker}

Early onset of alcohol and other drug use in adolescence is a marker of sustained problem use in adolescence as well as elevated risk for later AUD in adulthood (Grant and Dawson, 1997; Webb et al., 1991; White, 1992). Therefore, it is important to understand the antecedents of these behaviors as potentially important mediators of the etiologic pathway.

There is considerable evidence suggesting that some risk and protective factors for substance use can be identified early in life (Kellam et al., 1980; Masse and Tremblay, 1997; Zucker and Wong, 2005). However, much work remains to be carried out to identify the specific characteristics and manner of operation of these early antecedents. This study examined the developmental trajectories of 2 early childhood personality characteristics, behavioral control and resiliency, that have received significant attention as potentially core risk and protective factors for the emergence of early alcohol and other drug use. Behavioral control refers to the tendency to express or contain one's impulses, motor responses, and behaviors. Resiliency refers to the ability to adapt flexibly one's characteristic level of control in response to the environment. These 2 constructs have their origins in the seminal work of Block and Block (1980) on ego control and ego resiliency and are theoretically tied to the meticulous and comprehensive work of Eisenberg and colleagues on reactive control and resiliency (Eisenberg and Spinrad, 2004; Eisenberg et al., 2003). We use the terms "behavioral control" and "resiliency" instead of alternative terms to maintain consistency with the current substance abuse risk literature (National Institute on Alcohol Abuse and Alcoholism, 2000; Sher, 1991).

Prior studies have not examined the developmental trajectories of behavioral control and resiliency. Moreover, although there are data linking behavioral control and resiliency to drug use, no study has prospectively examined the effects of the 2 constructs on initial onset of alcohol use and alcohol-related problems (e.g., drunkenness, missing school because of drinking). Last, past studies rarely examined specificity of relations between the 2 constructs and particular forms of substance use. Do behavioral control and resiliency predict the use of certain drugs, or do the 2 constructs predict substance use in general? The current study attempts to address these questions.

Method. The study is part of the ongoing Michigan Longitudinal Study (Zucker and Fitzgerald, 1991; Zucker et al., 2000). Participants were 514 children of alcoholic individuals (COAs) and matched controls (365 boys, 149 girls; $74.9 \%$ COAs; $25.1 \%$ controls). Respondents were interviewed at 3-year intervals. The data presented in this paper involve the first 4 waves of the study. The children were 3 to 5 years old at Time 1, 6 to 8 years old at Time 2, 9 to 11 years old at Time 3, and 12 to 14 years old at Time 4 . Analyses required that all children have data on the substance use outcomes and at least one wave of data on behavioral control and resiliency. All families were Caucasian-American.

Behavioral control and resiliency (ages 3-14) were measured by interviewers' ratings using the California Child Qsort (CCQ). Alcohol and other illicit drug use (ages 12-17) was assessed by a questionnaire measure of frequency and quantity of alcohol use and problems, and frequency of other drug use and problems. Internalizing and externalizing problems (ages 12-14) were measured by maternal ratings of these behaviors on the Achenbach Child Behavior Rating Scale.

Owing to age variability within the same assessment waves, variability in the spacing of waves, and variability in the number of waves per respondent, we opted to (1) use age as the unit of time instead of wave and (2) treat time more flexibly by allowing the spacing and the number of measurements to vary across individuals in the analysis (Metha and West, 2000; Singer and Willet, 2003). The data were analyzed by a series of latent growth models with random times of measurement using MPLUS 3.0. In all analyses, the factor loadings in the least generalized models $(\Lambda)$ were allowed to vary across individuals.

Results and Discussion. Children varied in both the initial status and the rate of change of behavioral control. Behavioral control increased over time, as indicated by a significant latent slope factor. As children matured, they became less impulsive and more controlled. This is consistent with other developmental findings that children's self-control develops with age (Block et al., 1988; Kellam et al., 1980; Masse and Tremblay, 1997). Conversely, resiliency remained stable over time, as indicated by the nonsignificant means and variance of the latent slope factor. It is possible that individual differences in resiliency reflect a child's early temperament as well as the goodness of fit (i.e., compatibility) between the child's temperament and his or her environment. The lack of change in resiliency scores over time may imply that regulatory skills related to 
resiliency are rooted in early temperament and that this temperament is stable. Alternatively, it may suggest that the environment of the children in this study is stable and the environment creates the kind of stability observed in the data. However, as study participants approach late adolescence and have more independence from family, we may see more changes.

A curvilinear relationship between behavioral control and resiliency existed, such that extremely low and extremely high levels of behavioral control were associated with lower levels of resiliency. Children with lowest or highest levels of behavioral control were the least resilient in a developmental fashion. Children with moderately high levels of behavioral control were the most resilient. The relationship between behavioral control and resiliency was theoretically meaningful and consistent with the work of the Blocks and Eisenberg and colleagues (Block and Block, 1980; Eisenberg et al., 2000). To our knowledge, this is the first study to demonstrate how the 2 traits are related to one another longitudinally.

Controlling for the effects of parental alcoholism, age, and externalizing problems, behavioral control and resiliency predicted alcohol and drug use outcomes in meaningful ways. By adolescence, children with slower rates of increase in behavioral control over time were more likely to drink, to report having been drunk, to experience alcohol-related problems, and to have used illicit drugs other than alcohol by age 14 . This is a new finding that extends our understanding of how early personality characteristics may lead to early onset of drinking and illicit drug use. Although low behavioral control was also related to externalizing behavior problems, behavioral control predicted substance use even when externalizing problems were statistically controlled. This provides theoretical support for distinguishing the personality trait of behavioral control from the psychopathological construct of externalizing problems and shows that the trait carries important incremental predictive power from childhood into adolescence. Adolescents with higher initial levels of resiliency were less likely to start drinking by age 14 and less likely to have either internalizing or externalizing problems. However, resiliency did not predict the onset of drunkenness, alcohol-related problems, and the use of other illicit drugs in the presence of behavioral control.

Low behavioral control predicted all alcohol and drug use outcomes. In contrast, low resiliency predicted early onset of alcohol use only, but not other drug use. Both traits are theoretically related to self-regulation. The presence of regulatory skills and habits, or lack thereof, may be a crucial factor influencing important developmental outcomes in adolescence. Helping youth to develop such skills and habits may prove to be a promising intervention strategy for the prevention of substance abuse in adolescence.

The finding that behavioral control has a stronger relationship with drug use than resiliency is similar to the data reported by Block et al. (1988). These authors suggested that behavioral control is a key personality characteristic that leads to the values, personal susceptibilities, and the external circumstances associated with drug use in adolescence. Although low behavioral control may initially get some adolescents into trouble, it is the presence or absence of resiliency that determines whether adolescents work through and grow beyond their difficulties. According to this view, the deleterious effects of low resiliency will become apparent as individuals approach late adolescence and early adulthood.

Our results show that early childhood characteristics predict onset of alcohol and drug use 9 years later. Early onset of alcohol and drug use is associated with subsequent alcohol and drug abuse and dependence. These findings, together with other longitudinal studies linking behaviors in early childhood and alcohol/drug use in adolescence (e.g., Kellam et al., 1980; Block et al. 1988; Masse and Tremblay, 1997), suggest that substance abuse prevention programs beginning in early childhood may yield fruitful results (Zucker and Wong, 2005). If early childhood behaviors place individuals at risk for alcohol and drug use, then programs aiming at changing those behaviors may protect individuals from experimenting with drugs and alcohol. These programs might focus on teaching youngsters skills on delay of gratification, regulation of negative emotions and behaviors, as well as strategies related to attention focusing and distraction (i.e., diverting attention from unpleasant or task-irrelevant information) (Eisenberg and Spinrad, 2004).

\section{PARENT PSYCHOLOGICAL DYSREGULATION PREDICTS OFFSPRING ADOLESCENT ALCOHOL PROBLEMS}

\section{Duncan B. Clark and Jack R. Cornelius}

The children of parents with substance use disorders (SUDs), compared with reference children, have accelerated onset of alcohol problems in adolescence (Clark et al., 2005). While this pattern has traditionally been attributed to the transmission of liability specific to alcohol involvement, a growing body of evidence indicates that a more global transmitted liability may be involved. This more global liability, termed psychological dysregulation, has been defined as delayed or deficient development of behavioral, emotional, and cognitive regulation (Tarter et al., 1999). Children of parents with SUDs have increased rates of mental disorder reflective of psychological dysregulation, including disruptive behavior disorders (DBDs: conduct disorder, oppositional defiant disorder, and attention deficit hyperactivity disorder; Clark et al., 1997). Psychological dysregulation, reflected in these mental disorders or scale scores, prospectively predicts substance involvement and related problems in adolescence and complements parental SUDs and childhood alcohol and tobacco experi- 
mentation in predicting adolescent substance involvement (Clark et al., 1999, 2005).

Childhood mental disorders reflective of psychological dysregulation may be transmitted from parent to child in a developmentally specific fashion. We examined data from families participating in the Center for Education and Drug Abuse Research (CEDAR) with mixed effects ordinal regression analyses that controlled for the nested data structure (Clark et al., 2004). The predominant predictor of specific child mental disorders, including DBDs, was a history of the corresponding disorders in both parents. These results were consistent with a model in which parent-child transmission of a propensity toward psychological dysregulation was manifested in developmentally specific forms of childhood psychopathology. Following the rationale that childhood DBDs represent transmittable manifestations of psychological dysregulation, the preliminary analyses reported here examined the extent to which parental reports of their own childhood DBDs predicted adolescent alcohol problems in their children.

The subjects were 560 children ( 425 boys, 135 girls) recruited for CEDAR through their fathers in late childhood (mean age $11.4 \pm 0.9$ years). Each family contributed 1 index child. The recruited groups included children at high risk for SUDs $(n=266)$, having fathers with histories of Diagnostic and Statistical Manual (DSM)-III-R-defined drug use disorders (i.e., abuse or dependence involving illicit substances), and children at low risk (LAR; $n=294$ ), having fathers without SUDs or other major adulthood mental disorders. Data collected at the baseline assessment in late childhood (age 10-12 years) were used to determine risk characteristics and data from follow-up assessments in early adolescence-, middle adolescence-, and early adulthood-determined outcomes. Other analyses with these data have been reported previously (Clark et al., 2005).

Diagnoses were made by the American Psychiatric Association DSM-III-R, the most recent DSM edition when the study was initiated. For children and parents, information concerning childhood mental disorders, alcohol problems, and SUDs was gathered with standard semistructured interviews with some modifications made for this project (Clark et al., 2001). To limit the consideration of DBDs to early-onset types, parents were considered to have had DBDs only when an onset was reported age 12 years or younger. To combine childhood DBDs for father and mother, a 3-level variable was constructed, with 0 assigned to cases with neither parent reporting DBDs, 1 assigned to cases with only 1 parent reporting DBDs, and 2 assigned to cases with both parents DBDs (i.e., Parent DBD group). Parent AUDs were similarly categorized to create a 3-level variable (i.e., Parent AUD group). The outcome variable was the first age at which the offspring indicated an alcohol problem, a characteristic that can be reliably assessed in adolescent samples (Martin et al., 2000). Since some children had not completed all described outcome assessments, survival analysis was used to test multivariate models.

Among these 560 families, both parents had no history of childhood DBDs in 396 cases, the mother or father had a history of DBDs in 154 cases, and both parents had a history of DBDs in 10 cases. Additional details on the families may be found in Clark et al. (2005). Among 560 index children, 211 developed an alcohol problem by age 19. In a Cox regression model predicting alcohol problem onset, the Parent DBD group significantly predicted onset age (Wald $\chi^{2}=20.3, d f=2, p<0.001$ ), with significant additional variance attributable to gender (Wald $\chi^{2}=15.4$, $d f=2, p<0.001)$. Ethnic group and socioeconomic status did not significantly contribute to the model and were eliminated. Figure 1 presents an inverse survival plot providing the proportion of parent DBD group (i.e., neither parent, 1 parent only, both parents) with an alcohol problem by age in years. While the Parent AUD group predicted offspring alcohol problems (Wald $\chi^{2}=6.2, d f=2$, $p=0.045)$, the Parent AUD group did not account for significant variance (Wald $\chi^{2}=1.1, d f=2, p=0.6$ ) in a multivariate model including the Parent DBD group (Wald $\left.\chi^{2}=15.0, d f=2, p=0.001\right)$.

These results extended our previously reported finding that parental reports of their own childhood characteristics predicted similar disorders in their offspring (Clark et al., 2004). This analysis indicated that parental reports of their childhood DBD were superior to their report of adulthood AUDs in prospectively predicting offspring

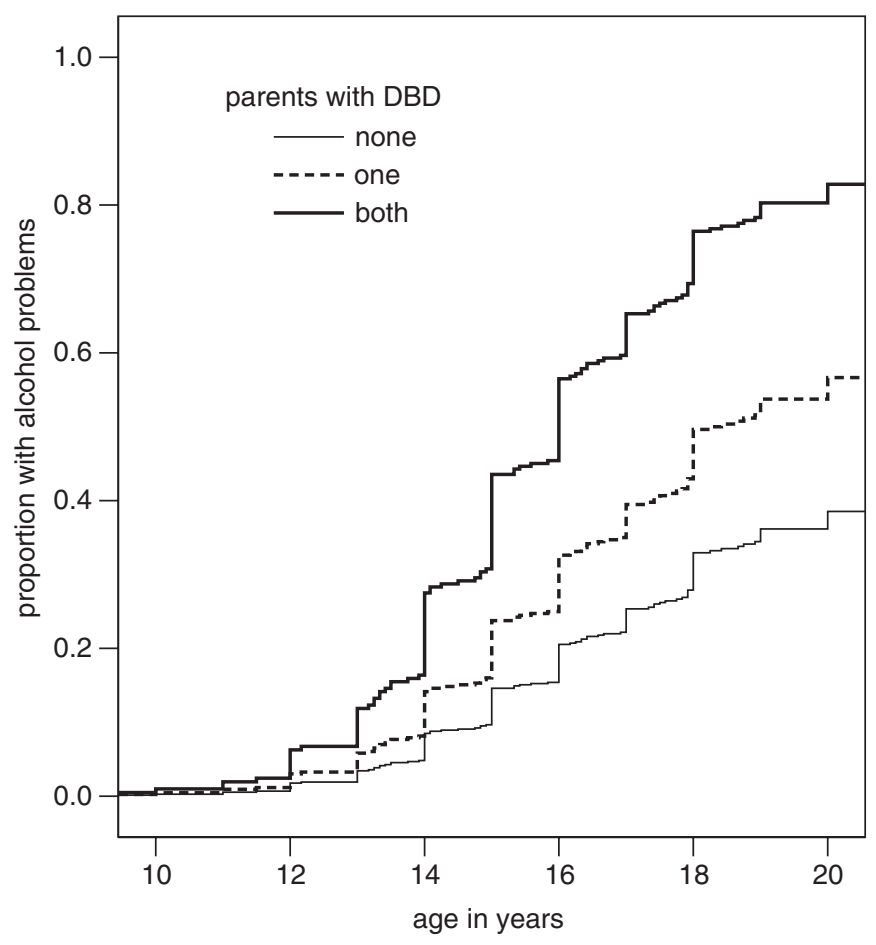

Fig. 1. Inverse survival plot for onset age of alcohol problems in offspring grouped by parent childhood disruptive behavior disorders. 
alcohol problems during adolescence. These results were consistent with the theoretical framework in which childhood DBDs are conceptualized as a manifestation of the trait of psychological dysregulation. Adulthood AUDs may result from a greater variety of influences and may be less directly relevant to predicting offspring characteristics during adolescence.

The study has several methodological limitations. The assessment of alcohol involvement used here was designed to be comprehensive and diachronic, i.e., continuous over time. Inquiries involving longer periods of retrospection yield less reliable variables. The reliability of alcohol involvement reports was maximized in this study by completing the initial assessment at an age prior to significant alcohol use, performing subsequent assessments at 2- to 3 -year intervals, and focusing on the first instance of an alcohol-related problem. Nevertheless, more frequent assessments would have been ideal. Another study limitation was that the extent to which the sample was representative of high-risk and low-risk populations of children was not testable. These analyses focused on the parents' manifestations of psychological dysregulation occurring prior to the birth of the offspring, suggesting nonenvironmental transmission consistent with a genetic hypothesis. The parents' adulthood manifestations of parental dysregulation, however, may be environmental influences (Moss et al., 1997). Additional studies with alternative designs and methods are needed to determine the mechanism through which parental psychological dysregulation influences offspring alcohol involvement.

Preventive programs are needed to disrupt the link between transmitted liabilities and adolescent alcohol and other drug involvement. Interventions focused on treatment for manifestations of psychological dysregulation such as DBDs may offer an effective strategy for preventing alcohol problems among high-risk children. While such interventions may seem more expensive than universally applicable strategies, the selective application of specialized preventive interventions optimized for children at high risk may prove to be a cost-effective approach.

\section{CHANGES IN ALCOHOL PROBLEMS OVER THE EARLY YEARS OF MARRIAGE: INDIVIDUAL, PARTNER, AND SOCIAL NETWORK INFLUENCES}

\section{Kenneth E. Leonard and Gregory G. Homish}

The extent of alcohol use and alcohol problems varies across the life span. As a consequence, researchers have applied probabilistic-developmental approaches to understanding the onset and trajectory of alcohol abuse and dependence. Zucker et al. (1995) stated this position "within such a framework ... one can conceive of risk as a fluid characteristic which increases or decreases depending upon the interplay of ongoing trajectory ... and the influence of new external and internal (stage triggered) causative agents." (p. 17). From this perspective, understanding the risks for alcohol dependence can be greatly informed by examining changes in drinking and drinking problems over specific developmental transitions, in particular, transitions that are associated with changes in drinking or drinking problems.

The current study focuses on the transition to marriage, a critical developmental transition, both generally and from an alcohol problems perspective. There is often a marked shift away from more individualistic values and toward more interdependent and socially positive values over this transition. There are also changes that involve the establishment of a mutually satisfactory relationship and the reestablishment or redefinition of ties, both as individuals and as a couple, with each member's extended family and peer network (Boss, 1983; McGoldrick and Carter, 1982). Marriage is also a vital transition with respect to alcohol use and alcohol problems, with significant reductions in drinking and drinking problems and shifts in drinking contexts over this period (Miller-Tutzauer et al., 1990; Roberts and Leonard, 1997; Zucker, 1979).

In this paper, we examined the development of alcohol problems among men and women without alcohol problems at marriage and the continuation/cessation of alcohol problems among men and women with alcohol problems at marriage. The study focused on 490 husbands and 531 wives participating in the Adult Development Study (ADS). The ADS is a longitudinal study of alcohol use and marriage and involved assessments at the time of marriage and at selected anniversaries. The study is currently completing assessments at the seventh anniversary, but the current study is based on the individuals in these couples who completed the premarital assessment and the assessment at the second anniversary. Participants were recruited at the city hall after they had applied for a marriage license. Only couples in which the marriage was the first for both husband and wife, the individuals were 18 years or older, both spoke English, and both were literate were eligible to participate. Husbands and wives who agreed to participate were given identical questionnaire packets to complete at home. Couple members were asked to complete the questionnaires independently at home within 3 weeks and to not discuss the questions or answers with their partner until after both had mailed back the materials. About the time of their anniversaries, couple members were mailed questionnaire packets similar to those they had completed at the time of marriage. Procedures were identical to those used at the first assessment.

At the time of marriage, the average ages [mean (SD)] of the 490 husbands and 531 wives were 28.0 (5.9) and 26.6 (5.5) years, respectively. A fairly large percentage of the sample was European American (husbands, 58\%; wives, $61 \%$ ). Approximately $29 \%$ of husbands and wives were African American. There were very small percentages of Hispanic, Asian, and Native American couples. Some college education or more had been completed by 61 and $67 \%$ of the husbands and wives, respectively. At marriage, $38 \%$ 
of husbands and $42 \%$ of the wives were parents. For both husbands and wives, we compared those with data for at both waves to individuals who did not have data at the second anniversary with respect to baseline sociodemographic and alcohol variables. There were no statistically significant differences between completers and dropouts.

There were a series of factors that have been associated with drinking in previous studies that were included in our analyses. We assessed alcohol problems with a series of questions that were modified from the National Alcohol Survey (Clark and Hilton, 1991), the Drinker Inventory of Consequences (Miller and Tonigan, 1995), and the work of Polich and Orvis (1979). We also assessed the frequency of heavy drinking and intoxication and the average daily consumption of alcohol. We also assessed a series of risk factors that have previously been linked to the development of heavy drinking, drinking problems, or alcohol dependence. These factors included a family history of alcoholism, antisocial behavior, depressive symptoms, alcohol expectancies, and relationship quality, peer drinking, and partner heavy drinking.

Based on alcohol problems at the time of marriage and at the second anniversary, we classified husbands and wives into 4 groups as follows: No Problems (no alcohol problems at either time), Persistent Problems (alcohol problems above the median at both times), Increasing Problems (no alcohol problems at marriage but alcohol problems above the median at the second anniversary), and Decreasing Problems (alcohol problems above the median at marriage but no alcohol problems at the second anniversary). The classification for wives identified $61 \%$ No Problems, 5\% Persistent Problems, 10\% Increasing Problems, and 20\% Decreasing Problems. For husbands, the classification identified $41 \%$ No Problems, $12 \%$ Persistent Problems, 12\% Increasing Problems, and 24\% Decreasing Problems.

We conducted repeated-measures analyses of covariance to examine the relationship of the risk factors with group status. Separate analyses were conducted for husbands and wives. We first compared the Persistent Problem and Decreasing Problems groups to determine factors related to continuation versus discontinuation of problems among individuals with premarital alcohol problems. Then, we compared the No Problem and the Increasing Problem groups to determine factors related to the development of alcohol problems after marriage.

Several factors differentiated the groups, either as main effects or in the context of group-by-time interactions. Group-by-time interactions were significant for positive alcohol expectancies for both the development and the continuation analyses for both husbands and wives. In general, groups with a riskier status (Increasing and Stable High) manifested more positive alcohol expectancies at the time of marriage than did groups with a less risky status (No Problems and Decreasing), and this difference increased over time.
Drinking among one's peer network was also an important factor, but the results were somewhat different across the development and continuation analyses for husbands and wives. There was a main effect of group in the continuation analyses for husbands, with stable high husbands having a larger proportion of heavy drinkers in their network in comparison with the decreasing group, a difference that was apparent at the premarital assessment and remained through the second anniversary. A similar finding was observed in the development analysis for wives, with Increasing Wives having a larger proportion of heavy drinkers in their network in comparison with the No Problem group. In the development analyses for husbands, there was a group-by-time interaction, indicating that the No Problem and Increasing group could not be distinguished from each other at the time of marriage. Over time, the Increasing group reported an increased number of heavy drinking peers, suggesting that this factor paralleled the development of alcohol problems.

Finally, the analyses suggested that women in the Increasing group had husbands with heavier drinking than did women in the No Problem group, suggesting that husband drinking has an impact on wife drinking problems. Interestingly, husbands in the Increasing group had wives who increased their drinking over time, suggesting that the drinking problems in the husbands in this group may have influenced their wives toward heavier drinking at the second anniversary.

This study revealed a high degree of stability of alcohol problems over the transition to marriage. Positive alcohol expectancies were related to alcohol problems after marriage for both men and women and appeared to operate similarly for both the development and continuation of alcohol problems. Characteristics of the social network, specifically the heavy drinking of peers and one's partner, operated differently for men and women and differently for the development and continuation of problems. Other factors that have been commonly associated with the development of alcoholism, such as family history and antisocial behavior, were not associated with changes in group status, suggesting that these factors may have had their impact prior to this developmental transition.

\section{MATURING OUT OR PARTYING ON: NATIONAL PROSPECTIVE DATA ON HOW THE COURSE OF HEAVY DRINKING DURING LATE ADOLESCENCE AND EARLY ADULTHOOD RELATES TO AUDS IN MIDDLE ADULTHOOD}

\section{John E. Schulenberg, Alicia Merline, and Patrick M. O'Malley}

Excessive drinking during late adolescence and early adulthood is a likely necessary condition for alcohol (and other drug) difficulties in middle adulthood. Nevertheless, such earlier excessive drinking is not a sufficient condition for later difficulties with alcohol. Indeed, many individuals 
mature out of heavy drinking patterns of late adolescence/ early adulthood and move on with their lives avoiding later difficulties with alcohol (and other drugs). Thus, a central question is to what extent does heavy drinking in late adolescence and early adulthood predict difficulties with alcohol in middle adulthood? But answering this and related questions about the long-term developmental connections has been limited by methodological difficulties. In addition to limited long-term prospective data, the predominant strategy to understanding such connections has been to focus on the level of substance use at one point and examine its relation to use and abuse at a later point in time, a strategy that fails to recognize the complex patterns of stability and change in substance use during late adolescence and early adulthood (Schulenberg et al., 2003). The present paper attempts to overcome some of the previous limitations by focusing on national panel data from the Monitoring the Future study (Johnston et al., 2005) and emphasizing individual trajectories of stability and change in heavy alcohol use during the transition to adulthood. In particular, our purpose is to examine the relationship between heavy drinking during late adolescence/ early adulthood (ages 18-26) and age 35 AUDs. There are 3 phases of the analyses in terms of predicting age 35 AUDs as follows: (1) examine heavy drinking as static latent predictors ages 18 to 26; (2) examine heavy drinking as intercept and slope latent predictors across ages 18 to 26; and (3) examine heavy drinking as latent trajectory class predictors across ages 18 to 26 . Because the experiences of late adolescence and early adulthood vary by college attendance and gender, we considered both of these as potential moderators in all phases of the analyses.

\section{Method}

Data come from the Monitoring the Future study, an NIDA-funded project on epidemiology and etiology of substance use among adolescents and young adults. The study includes nationally representative samples of high school seniors $(\sim 16,000 /$ year $)$ as well as a panel component following 2,400 randomly selected respondents from each graduating class into young adulthood (to age 30 ) on a biennial basis. Follow-up surveys into middle adulthood begin at age 35. In the present analyses, we include 6 waves of panel data (ages 18, 20, 22, 24, 26, and 35) from the 1976 to 1985 senior year cohorts (approximate $N$ of 12,000). Heavy drinking was assessed at each time of measurement on the frequency of having 5 or more drinks in a row in the previous 2 weeks. AUDs were assessed at age 35 with 19 items on symptoms experienced over the past 5 years including both alcohol abuse (e.g., "hurt your relationship with spouse, girlfriend/boyfriend") and dependence ("needed more for same effect"); the items are based on DSM content, similar to items used in the National Health Interview Survey (e.g., Muthen, 1995). Both gender and college status (enrolled full-time at a 4-year university at age 20) were examined as potential moderators.

\section{Results}

Phase 1. We first examined heavy drinking at ages 18 , $20,22,24$, and 26 separately as predictors of AUDs at age 35 (using latent variable modeling in LISREL 8; we estimated $20 \%$ error variance in the predictors). Based on these 5 analyses, standardized regression coefficients were $0.34,0.31,0.43,0.38$, and 0.43 , respectively. Clearly, even at age 18 , heavy drinking is fairly strongly predictive of age 35 AUDs, accounting for nearly $12 \%$ of its variance. The predictive power increased with age (although not entirely progressively), with age 26 heavy drinking accounting for nearly $19 \%$ of the variance in age 35 AUDs. We repeated these analyses for each of 4 subgroups as follows: noncollege males and females and college males and females. For the most part, results were similar to those found for the total sample, although heavy drinking at all ages was most predictive of age 35 AUD for noncollege males and least predictive for college females. Overall, heavy drinking at any one point in time during late adolescence and early adulthood is fairly predictive of AUDs at the start of middle age, and not surprisingly, this predictive power tends to increase with age (although not entirely progressively). Particularly noteworthy is that heavy drinking is most predictive of later AUDs for men who do not attend college following high school and least predictive for women who do attend college, suggesting that for college women, heavy drinking tends to be more experimental and short-lived.

Phase 2. In the second phase of the analyses, we considered the course of heavy drinking between ages 18 and 26 and its impact on age 35 AUDs. Latent curve modeling analyses (using LISREL 8) were conducted, which included an intercept, a linear slope, and a quadratic slope (when needed) to represent the average trajectory of heavy drinking and individual deviations from the average trajectory. In the total sample, the heavy drinking latent intercept and linear slope adequately represented the average course of heavy drinking, with significant variation in both indicating individual differences in level and course. The latent linear slope was negative, indicating that on average, heavy drinking declined between ages 18 and 26. Both of these latent variables significantly predicted age 35 AUDs (standardized regression coefficients were 0.55 for the intercept and 0.37 for the linear slope), indicating that initially higher levels and slower declines over time in heavy drinking were associated with higher rates of age 35 AUDs. Together, the latent intercept and linear slope accounted for nearly $30 \%$ of the variance in age 35 AUDs, suggesting the advantage of focusing on heavy drinking in terms of trajectories rather than simply a single point in time (as was done in Phase 1). In considering the gender by college status subgroups, the intercept and linear slope 
were adequate for noncollege males and females, and together, the 2 latent variables accounted for 45 and 17\% of the variance in age 35 AUDs for males and females, respectively. For college males and females, it was necessary to include a latent quadratic slope as well to represent the increase and decrease in heavy drinking across the ages - indeed, a more rapid decrease was associated with a lower likelihood of age 35 AUDs. Together, the 3 latent variables accounted for 18 and $14 \%$ of the variance in age 35 AUDs for college males and females, respectively. Overall, the findings indicate that the higher the initial level and the slower the decrease in heavy drinking during late adolescence and early adulthood, the more likely one experiences AUDs at age 35. Based on the variance accounted for in age 35 AUDs, the trajectory of heavy drinking provides a better prediction of later AUDs than does heavy drinking at single points in time during this period. Consistent with what was found in Phase 1, heavy drinking is most predictive of later AUDs for noncollege males and least predictive for college females.

Phase 3. In the third phase of analyses, we again focused on the trajectory of heavy drinking to predict age 35 AUDs, but instead considered different trajectory forms, grouping individuals according to how their heavy drinking changed over time. We used growth mixture modeling (via MPLUS) to categorize individuals into 1 of 5 classes based on their pattern of heavy drinking from age 18 to age 26 as follows (classes are consistent with previous analyses using different approaches - e.g., Schulenberg et al, 1996): Chronic heavy drinkers ( $8 \%$ of the sample) drank frequently from age 18 through age 26, Increased drinkers $(9 \%)$ drank infrequently at age 18 and frequently by age 26 , Decreased drinkers $(14 \%)$ frequently drank heavily at age 18 , but rarely drank heavily by age 26 , Fling drinkers $(5 \%)$ rarely drank heavily at ages 18 and 26, but reported frequent heavy drinking around ages 20 through 24 , and Never/Rare drinkers $(64 \%)$ reported little to no heavy drinking between the ages of 18 and 26. Based on a comparison of age 35 AUDs across these 5 groups (using ANOVAs), we found that the Chronic and Increased groups had significantly higher AUD scores than the others and that the Decreased and Fling groups had significantly higher AUD scores than the Never/Rare group. In considering the college status by gender subgroups, we found that the 5 trajectory groups were present in each subgroup, although with different prevalences (e.g., noncollege males were overrepresented in the Chronic group, college males were overrepresented in the Fling group, and females were overrepresented in the Never/Rare group). For variation in age 35 AUDs as a function of trajectory group, the findings for the college status by gender subgroups were very similar to those for the total sample. Thus, overall, those who continue at a high level or increased trajectory of heavy drinking during into early adulthood are most likely to experience AUDs at age 35, whereas those who decrease their heavy drinking during this time (regardless of how much they were drinking) are less likely to experience later AUDs.

\section{Conclusions}

Clearly, heavy drinking during late adolescence and early adulthood is related to later difficulties with alcohol, but attempting this prediction with heavy drinking at one point in time is less successful than if we consider the course of heavy drinking across time. This is especially true during late adolescence and early adulthood when heavy drinking is more common and less prognostic of ongoing use of and difficulties with alcohol. Of particular importance, heavy drinking during late adolescence and early adulthood is most predictive of AUDs in middle adulthood for men who do not go on to college after high school and least predictive for women who do attend college.

\section{DISCUSSION FOR THE SYMPOSIUM}

\section{Robert A. Zucker}

Together, these presentations reiterate a state of the science that is not well known to the alcohol research and treatment communities, but that has been increasingly evident in the literature over the past generation, namely, that robust indicators of longer term problem drinking outcomes are identifiable considerably earlier than the time of the clinical event (see Zucker and Wong, 2005). The Wong and Clark papers, both using high-risk designs, continue to show that measures of psychological dysregulation are precursive to two of the best proxies we have for estimating AUD, namely, early onset of drinking and drinking problems. Moreover, these harbinger indicators, one involving stable personality traits, the other a familial indicator of disruptive behavior, are detectable 10 to 19 years prior to the problem drinking marker. The specific vulnerability indicators, behavioral undercontrol, low resiliency, and a diathesis for disruptive behavior, all share a common thread involving deficits in the regulation of cognition, behavior, and emotion. These are individual difference characteristics that are nonspecific to alcohol and other drug use; that is, the presence of the risk heightens the probability of a substance abusing outcome but the factor also indicates riskiness for other symptomatic behaviors, including delinquency, emotionality, depression, etc. In this regard, these behavioral findings are consistent with the genetic literature indicating that well more than half of the heritable risk for substance use disorders is non-drug specific (Kendler et al., 2003; Tsuang et al., 1998).

These dsyregulation/undercontrol indicators also differ in important ways that remain to be understood. The fact that behavioral undercontrol has effects above and beyond externalizing and that the linkages among the cognitive, behavioral, and emotional indicators of psychological dysregulatory liability are of relatively low order suggest that 
multiple facets of risk are still operative here and remain to be identified.

The latter 2 papers demonstrate that prospective prediction of risky drinking outcomes is effective even across times of social discontinuity, such as marriage (Leonard and Homish) and movement from late adolescence to middle adulthood (Schulenberg and colleagues). The studies also indicate that drinking-specific variance (level of drinking at point of marriage, positive expectancies about alcohol's effects, involvement in a heavy drinking peer network, being married to a heavy drinking husband; in the Leonard and Homish study, frequency of binge drinking in the Monitoring the Future study) is likewise a robust predictor of later drinking outcomes. The Leonard and Homish data indicate that both cognitions about alcohol (baseline positive expectancies about drinking) and social experience (involvement with a drinking peer network) sustain heavy drinking and in some instances contribute to change. The pattern of such effects was not the same for men and women, suggesting again that the drinking worlds for the 2 genders are different. The MTF data, extending 17 years over early to middle adulthood, also indicate that this is the case (college women's binge drinking is least predictive of their later AUD and most predictive for noncollege men).

The Schulenberg et al. analyses also compellingly demonstrate that understanding the history of use, or alternatively, the trajectory of use, provides a much more powerful indicator of long-term outcome than does the single assessment marker at one developmental waypoint. In practical terms, this suggests that clinical diagnosis should attempt to incorporate indicators of level of problem at some developmentally appropriate baseline point (intercept), rate of change (or not) in use over time (slope), and variation in pattern of use over time (as indexed by curvilinearity on the one hand, or class membership on the other) to more effectively anticipate later AUD outcome.

The work presented here reflects a sea change in the portrayal of the emergence of risk and later alcohol problems as a dynamic, not necessarily stable, process over time, requiring a new vocabulary of trajectory variation to characterize it. The papers also drive home that the matrix of risk is not yet an integrated one; as it becomes better differentiated, our ability to foretell future drinking outcomes should only improve.

\section{REFERENCES}

Block JH, Block J (1980) The role of ego-control and ego-resiliency in the organization of behavior. in Minnesota Symposia on Child Psychology, Vol. 6 (Collins WA ed), pp 39-101. Erlbaum, Hillsdale, NJ.

Block J, Block JH, Keyes S (1988) Longitudinally foretelling drug usage in adolescence: early childhood personality and environmental precursors. Child Dev 59:336-355.

Boss PG (1983) The marital relationship: boundaries and ambiguities. in Stress and the Family: Vol 1. Coping with Normative Transitions (McCubbin HI, Figley CR eds), pp 26-40. Brunner/Mazel, New York.
Clark DB (2004) The natural history of adolescent alcohol use disorders. Addiction 99:5-22.

Clark DB, Cornelius JR, Kirisci L, Tarter RE (2005) Childhood risk categories for adolescent substance involvement: a general liability typology. Drug Alcohol Depend 77:13-21.

Clark DB, Cornelius JR, Wood DS, Vanyukov M (2004) Psychopathology risk transmission in children of parents with substance use disorders. Am J Psychiatry 161:685-691.

Clark WB, Hilton ME (1991) Alcohol in America: Drinking Practices and Problems. State University of New York Press, Albany, NY.

Clark DB, Moss H, Kirisci L, Mezzich AC, Miles R, Ott P (1997) Psychopathology in preadolescent sons of substance abusers. J Am Acad Child Adol Psychiatry 36:495-502.

Clark DB, Parker AM, Lynch KG (1999) Psychopathology, substance use and substance related problems. J Clin Child Psychol 28:333-341.

Clark DB, Pollock NA, Mezzich A, Cornelius J, Martin C (2001) Diachronic assessment and the emergence of substance use disorders. J Child Adol Subs Abuse 10:13-22.

Clark DB, Winters KC (2002) Measuring risks and outcomes in substance use disorders prevention research. J Consult Clin Psychol 70:1207-1223

Eisenberg N, Fabes RA, Guthrie IK, Reiser M (2000) Dispositional emotionality and regulation: their role in predicting quality of social functioning. J Person Soc Psychol 78:136-157.

Eisenberg N, Spinrad TL (2004) Emotion-related regulation: sharpening the definition. Child Dev 75:334-339.

Eisenberg N, Zhou Q, Losoya SH, Fabes RA, Shepard SA, Murphy BC, Reiser M, Guthrie IK, Cumberland A (2003) The relations of parenting, effortful control, and ego control to children's emotional expressivity. Child Dev 74:875-895.

Grant BF, Dawson DA (1997) Age at onset of alcohol use and its association with DSM-IV alcohol abuse dependence: results from the National Longitudinal Alcohol Epidemiologic Survey. J Subst Abuse 9:103-110.

Johnston LD, O'Malley PM, Bachman JG, Schulenberg JE (2004) Monitoring the Future National Survey Results on Drug Use, 1975-2003. Volume I: Secondary School Students. (NIH Pub No. 04-5507). National Institute on Drug Abuse, Bethesda, MD.

Kellam SG, Ensminger ME, Simon MB (1980) Mental health in first grad and teenage drug, alcohol, and cigarette use. Drug Alcohol Depend 5:273-304.

Kendler KS, Prescott CA, Myers J, Neale MC (2003) The structure of genetic and environmental risk factors for common psychiatric and substance use disorders in men and women. Arch Gen Psychiatry 60:929-937.

Martin CS, Pollock NK, Bukstein OG, Lynch KG (2000) Inter-rater reliability of the SCID alcohol and substance use disorders section among adolescents. Drug Alcohol Depend 59:173-176.

Masse LC, Tremblay RE (1997) Behavior of boys in kindergarten and the onset of Substance use during adolescence. Arch Gen Psychiatry 54:62-68.

McGoldrick M, Carter EA (1982) The family life cycle. in Normal Family Process (Walsh F ed), pp 167-195. Guilford Press, New York.

Metha PD, West SG (2000) Putting the individual back into individual growth curves. Psychol Methods 5:23-43.

Miller WR, Tonigan JS (1995) The Drinker Inventory of Consequences (DrInC). An Instrument for Assessing Adverse Consequences of Alcohol Abuse, Test Manual. NIAAA, Rockville, MD.

Miller-Tutzauer C, Leonard KE, Windle M (1990) Marriage and alcohol use: a longitudinal study of maturing out. J Stud Alcohol 52:434-440.

Moss HB, Clark DB, Kirisci L (1997) Developmental timing of paternal substance use disorder offset and the severity of problem behaviors in their prepubertal sons. Am J Addict 6:30-37.

Muthen BO (1995) Factor analysis of alcohol abuse and dependence symptom items in the 1988 National Health Interview survey. Addiction 90:637-645. 
National Institute of Alcohol Abuse and Alcoholism (2000) 10th Special Report to the U.S. Congress on Alcohol and Health. U.S. Department of Health and Human Services, Washington, DC.

Polich JM, Orvis BR (1979) Alcohol Problems: Patterns and Prevalence in the US Air Force. Rand, Santa Monica, CA.

Roberts LJ, Leonard KE (1997) Gender differences and similarities in the alcohol and marriage relationship. in Gender and Alcohol (Wilsnack S, Wilsnack R eds), pp 289-311. Rutgers Center of Alcohol Studies, Piscataway, NJ.

Schulenberg JE, Maggs JM, O'Malley PM (2003) How and why the understanding of developmental continuity and discontinuity is important: the sample case of long-term consequences of adolescent substance us. in Handbook of the Life Course (Mortimer JT, Shanahan MJ eds), pp 413-436. Plenum Publishers, New York.

Schulenberg JE, O’Malley PM, Bachman JG, Wadsworth KN, Johnston LD (1996) Getting drunk and growing up: trajectories of frequent binge drinking during the transition to young adulthood. J Stud Alcohol 57:289-304.

Sher KJ (1991) Children of Alcoholics: A Critical Appraisal of Theory and Research. University of Chicago Press, Chicago.

Singer JD, Willet JB (2003) A framework for investigating change over time. in Applied Longitudinal Data Analysis: Modeling Change and Event Occurrence (Singer JD, Willet JB eds), pp 3-15. Oxford University Press, New York.

Tarter R, Vanyukov M, Giancola P, Dawes M, Blackson T, Mezzich A, Clark DB (1999) Epigenetic model of substance use disorder etiology. Dev Psychopathol 11:657-683.

Tsuang MT, Lyons MJ, Meyer JM, Doyle T, Eisen SA, Goldberg J, et al (1998) Co-occurrence of abuse of different drugs in men. Arch Gen Psychiatry 55:967-972.
Webb JA, Baer PE, Caid CD, McLaughlin RJ, McKelvey RS (1991) Concurrent and longitudinal assessment of risk for alcohol use among seventh graders. J Early Adol 11:450-465.

White HR (1992) Early problem behavior and later drug problems. J Res Crime Delinquency 29:412-429.

Zucker RA (1979) Developmental aspects of drinking through the young adult years. in Youth, Alcohol, and Social Policy (Blane HT, Chafetz ME eds), pp 91-146. Plenum Press, New York.

Zucker RA (1994) Pathways to alcohol problems and alcoholism: a developmental account of the evidence for multiple alcoholisms and contextual contributions to risk. in The Development of Alcohol Problems: Exploring the Biopsychosocial Matrix of Risk (NIAAA Research Monograph 26, NIH Publication No. 94-3495), pp 255-289. US Department of Health and Human Services, Rockville, MD.

Zucker RA, Fitzgerald HE (1991) Early developmental factors and risk for alcohol problems. Alcohol Health Res World 15:18-24.

Zucker RA, Fitzgerald HE, Moses HD (1995) Emergence of alcohol problems and the several alcoholisms: a developmental perspective on etiologic theory and life course trajectory. in Developmental Psychopathology: Vol. 2, Risk, Disorder and Adaptation (Cicchetti D, Cohen DJ eds), pp 677-711. Wiley, New York.

Zucker RA, Fitzgerald HE, Refior SK, Puttler LI, Pallas DM, Ellis DA (2000) The clinical and social ecology of childhood for children of alcoholics: description of a study and implications for a differentiated social policy. in Children of Addiction: Research, Health, and Policy Issues (Fitzgerald HE, Lester BM, Zuckerman BS eds), pp 109-141. RutledgeFalmer, New York.

Zucker RA, Wong MM (2005) Prevention for children of alcoholics and other high risk groups. in Recent Developments in Alcoholism, Vol. XVII: Alcohol Problems in Adolescents and Young Adult (Galanter M ed), pp 299-319. Kluwer Academic/Plenum, New York. 